\title{
Impact parameter dependence of collective flow and its disappearance for different mass asymmetries.
}

\author{
Supriya Goyal \\ Department of Physics, Panjab University, Chandigarh 160 014, India \\ E-mail: ashuphysics@gmail.com
}

\begin{abstract}
We study the role of impact parameter on the collective flow and its disappearance for different mass asymmetric reactions. The mass asymmetry is varied from 0 to 0.7 keeping the total mass of the system fixed. Our results clearly indicate a significant role of impact parameter on the collective flow and its disappearance for the mass asymmetric reactions. The impact parameter dependence is also found to vary with mass asymmetry of the reaction.
\end{abstract}

\section{Introduction}

On the collision of two nuclei, the density and pressure increases in the interaction region and at finite colliding geometries, there is an inherent asymmetry in the pressure which results in a collective transverse flow of matter toward the direction of lowest pressure. This collective transverse flow is one of the most extensively used observable to study the equation of state (EoS) as well as in-medium nucleon-nucleon (nn) cross-section of the nuclear matter. At the particular value of energy where the attractive scattering (dominant at energies around $10 \mathrm{MeV} /$ nucleon) balances the repulsive interactions (dominant at energies around $400 \mathrm{MeV} /$ nucleon), collective transverse flow in the reaction plane disappears. This energy is termed as Balance Energy $\left(E_{b a l}\right)$ 1, 2, 3, 4, 5, 6, 7, 8, 9, 10.

Both collective flow and $E_{b a l}$ are found to be highly sensitive towards the nn cross-section [2, 3, 4, 5, 6, 7, 8, 9, 10, size of the system $\left(A_{T O T}=A_{T}+A_{P}\right.$; where $A_{T}$ and $A_{P}$ are the masses of the target and projectile, respectively) [4, mass asymmetry of the reaction $\left(\eta=\frac{A_{T}-A_{P}}{A_{T}+A_{P}}\right)[11$, nuclear matter equation of state [2, 3, 4, 5, 6, 7, 8, 9, 10, incident energy (E MeV/nucleon) [12] as well as the colliding geometry $\left(\hat{b}=\frac{b}{b_{\max }}\right.$; where $b_{\max }=R_{1}+R_{2} ; R_{i}$ is the radius of projectile or target) [2, 5, 7, 9, 10. Several experimental and theoretical attempts have been employed in order to explain and understand these observations [1, 2, 3, 4, 5, 6, 7, 8, 9, 10, 11, 12. Due to the decrease in the compression reached in heavy-ion collisions with increase in the impact parameter, $E_{b a l}$ is found to increase approximately linearly as a function of the impact parameter [13, 14]. But most of the studies in the literature mainly focus on the symmetric and nearly symmetric reactions. Our present aim, therefore, is at least twofold. (1) To study the variation of collective transverse flow with impact parameter for different $\eta$ keeping the incident energy fixed. (2) To study the sensitivity of collective transverse flow and its disappearance on the mass asymmetry of the reaction at different impact parameters. The Quantum Molecular Dynamics (QMD) model [8, 15, 16] used for the present analysis is explained in the section 2. Results and discussion are presented in section 3 followed by summary in section 4 . 


\section{Description of the model}

In the QMD model, each nucleon propagates under the influence of mutual two- and three-body interactions. The propagation is governed by the classical equations of motion:

$$
\begin{gathered}
\frac{d \vec{r}_{i}}{d t}=\frac{d H}{d \vec{p}_{i}}, \\
\frac{d \vec{p}_{i}}{d t}=-\frac{d H}{d \vec{r}_{i}},
\end{gathered}
$$

where the Hamiltonian is given by

$$
H=\sum_{i} \frac{\vec{p}_{i}^{2}}{2 m_{i}}+V^{t o t}
$$

Our total interaction potential $V^{\text {tot }}$ reads as [8, 15, 16]

$$
V^{t o t}=V^{L o c}+V^{Y u k}+V^{C o u l},
$$

with

$$
\begin{gathered}
V^{L o c}=t_{1} \delta\left(\vec{r}_{i}-\vec{r}_{j}\right)+t_{2} \delta\left(\vec{r}_{i}-\vec{r}_{j}\right) \delta\left(\vec{r}_{i}-\vec{r}_{k}\right), \\
V^{Y u k}=t_{3} e^{-\left|\vec{r}_{i}-\vec{r}_{j}\right| / m} /\left(\left|\vec{r}_{i}-\vec{r}_{j}\right| / m\right),
\end{gathered}
$$

with $m=1.5 \mathrm{fm}$ and $t_{3}=-6.66 \mathrm{MeV}$.

The static (local) Skyrme interaction can further be parametrized as:

$$
U^{L o c}=\alpha\left(\frac{\rho}{\rho_{o}}\right)+\beta\left(\frac{\rho}{\rho_{o}}\right)^{\gamma} .
$$

Here $\alpha, \beta$ and $\gamma$ are the parameters that define equation of state. The parameters $\alpha, \beta$, and $\gamma$ in above Eq. (7) must be adjusted so as to reproduce the ground state properties of the nuclear matter. The set of parameters corresponding to different equations of state can be found in Ref. [16]. It is worth mentioning that as shown by Puri and coworkers, Skyrme forces are very successful in the analysis of low energy phenomena such as fusion, fission and cluster-radioactivity, where nuclear potential plays an important role [17.

\section{Results and Discussion}

For the present study, we simulated the reactions of ${ }_{10}^{20} N e+{ }_{10}^{20} N e(\eta=0),{ }_{8}^{17} \mathrm{O}+{ }_{11}^{23} \mathrm{Na}(\eta=0.1)$, ${ }_{7}^{14} N+{ }_{12}^{26} M g(\eta=0.3),{ }_{5}^{10} B+{ }_{14}^{30} S i(\eta=0.5)$, and ${ }_{3}^{6} L i+{ }_{16}^{34} S(\eta=0.7)$ for total mass $\left(A_{T O T}\right)=40$, ${ }_{20}^{40} \mathrm{Ca}+{ }_{20}^{40} \mathrm{Ca}(\eta=0),{ }_{18}^{36} \mathrm{Ar}+{ }_{20}^{44} \mathrm{Ca}(\eta=0.1),{ }_{14}^{28} S i+{ }_{24}^{52} \mathrm{Cr}(\eta=0.3),{ }_{10}^{20} \mathrm{Ne}+{ }_{28}^{60} \mathrm{Ni}(\eta=0.5)$, and ${ }_{5}^{10} B+{ }_{32}^{70} G e(\eta=0.7)$ for total mass $\left(A_{T O T}\right)=80,{ }_{36}^{80} K r+{ }_{36}^{80} K r(\eta=0),{ }_{32}^{70} G e+{ }_{40}^{90} Z r(\eta=0.1)$, ${ }_{26}^{54} \mathrm{Fe}+{ }_{48}^{106} \mathrm{Cd}(\eta=0.3),{ }_{20}^{40} \mathrm{Ca}+{ }_{52}^{120} \mathrm{Te}(\eta=0.5)$, and ${ }_{12}^{24} \mathrm{Mg}+{ }_{58}^{136} \mathrm{Ce}(\eta=0.7)$ for total mass $\left(A_{\text {TOT }}\right)=160$, and ${ }_{52}^{120} \mathrm{Te}+{ }_{52}^{120} \mathrm{Te}(\eta=0),{ }_{48}^{108} \mathrm{Cd}+{ }_{56}^{132} \mathrm{Ba}(\eta=0.1),{ }_{38}^{84} \mathrm{Sr}+{ }_{66}^{156} \mathrm{Dy}(\eta=0.3)$, ${ }_{28}^{60} \mathrm{Ni}+{ }_{74}^{180} \mathrm{~W}(\eta=0.5)$, and ${ }_{18}^{36} \mathrm{Ar}+{ }_{82}^{204} \mathrm{~Pb}(\eta=0.7)$ for total mass $\left(A_{T O T}\right)=240$. The impact parameter is varied from $\mathrm{b} / \mathrm{b}_{\max }=0$ to 1 in small steps of 0.25 . The charges are chosen in a way so that colliding nuclei are stable nuclides. A soft equation of state with isotropic energy dependent cugnon cross-section (labeled as Soft ${ }^{i s o}$ ) is used for the present calculations.

The balance energy $\left(E_{b a l}\right)$ is calculated using the directed transverse momentum $\left\langle P_{x}^{\text {dir }}\right\rangle$, which is defined as:

$$
\left\langle P_{x}^{d i r}\right\rangle=\frac{1}{A} \sum_{i} \operatorname{sign}\{Y(i)\} \mathbf{p}_{x}(i)
$$



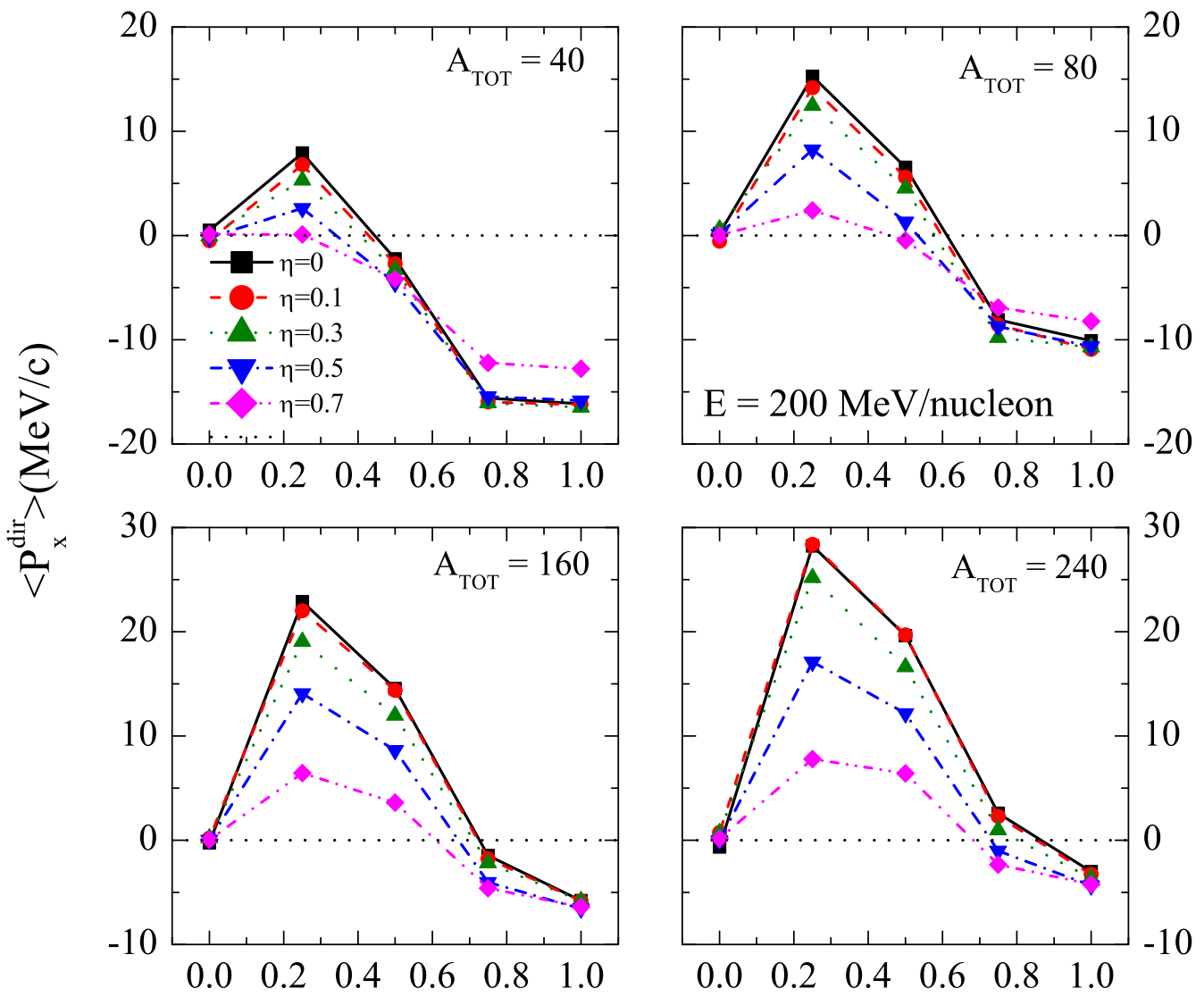

$\mathrm{b} / \mathrm{b}_{\max }$

Figure 1. (Color online) The $<P_{x}^{d i r}>(\mathrm{MeV} / \mathrm{c})$ as a function of reduced impact parameter $\left(b / b_{\max }\right)$ for different system masses. The results for different mass asymmetries $\eta=0,0.1$, $0.3,0.5$, and 0.7 are represented, respectively, by the solid squares, circles, triangles, inverted triangles, and diamonds. Results are at an incident energy of $200 \mathrm{MeV} /$ nucleon.

where $Y(i)$ and $\mathbf{p}_{x}(i)$ are the rapidity distribution and transverse momentum of $i^{\text {th }}$ particle, respectively.

In Fig. 1, we display at a fixed energy, the $\left\langle P_{x}^{d i r}\right\rangle$ as a function of reduced impact parameter $\left(b / b_{\max }\right)$ for $\eta=0-0.7$, keeping total system mass fixed as $40,80,160$, and 240 . All reactions are followed till $200 \mathrm{fm} / \mathrm{c}$, where $\left\langle P_{x}^{\text {dir }}\right\rangle$ saturates. In all cases $\left\langle P_{x}^{d i r}\right\rangle$ first increases with increase in impact parameter, reaches a maximal value and after passing through a zero at some intermediate value of impact parameter, attains negative values. The trend is uniform throughout the mass asymmetry range. The value of impact parameter at which $<P_{x}^{d i r}>$ attains a zero (which is termed as Geometry of Vanishing Flow (GVF) [18]) varies with $\eta$ and $A_{T O T}$. For lighter systems and larger $\eta$, the value of GVF is smaller compared to the heavier systems and smaller $\eta$.

In Fig. 2, the variation of GVF as a function of $\eta$ is displayed for different system masses. The percentage variation in GVF while going from $\eta=0$ to 0.7 is $-40.9 \%,-24.59 \%,-16.44 \%$, and $-20.93 \%$, respectively for $A_{T O T}=40,80,160$, and 240 . It is clear from the figure that the effect of mass asymmetry of the reaction on GVF decreases with increase in system mass. This 


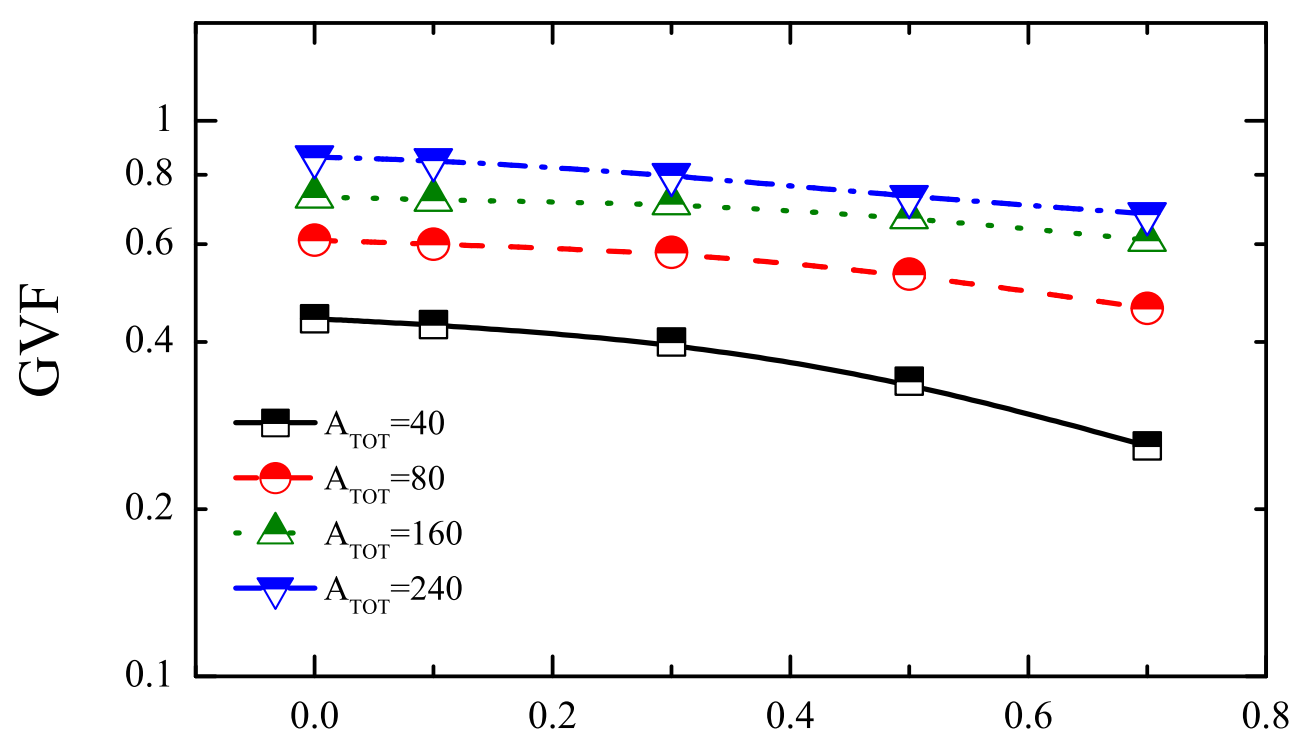

$\eta$

Figure 2. (Color online) The geometry of vanishing flow (GVF) as a function of $\eta$ for different system masses. The results for different system masses $\left(A_{T O T}\right)=40,80,160$, and 240 are represented, respectively, by the half filled squares, circles, triangles, and inverted triangles.

is similar to as predicted for $E_{b a l}$ [11]. Due to the decrease in nn collisions with increase in $\eta$ and impact parameter and increase in Coulomb repulsion with increase in $A_{T O T}$, the $E_{b a l}$ increases with increase in $\eta$ and impact parameter, while it decreases with increase in $A_{T O T}$. Since the present study is at fixed incident energy, therefore, the value of impact parameter, where flow vanishes, decreases as $\eta$ increases.

In Fig. 3, we display $\left\langle P_{x}^{d i r}>\right.$ as a function of incident energy ranging between 40 $\mathrm{MeV} /$ nucleon and $800 \mathrm{MeV} /$ nucleon, for different mass asymmetric reactions keeping the total mass of the system fixed as 240 . The results are shown for different impact parameters. In all the cases, transverse momentum is negative at lower incident energies which turns positive at relatively higher incident energies. The value of the abscissa at zero value of $\left\langle P_{x}^{d i r}\right\rangle$ corresponds to the balance energy. The figure indicates that (i) for all values of $\eta$ and impact parameters, the transverse momentum increases monotonically with increase in the incident energy. The increase is sharp at smaller incident energies compared to higher incident energies where it starts saturating. (ii) due to decrease in overlap volume and hence number of collisions with increase in $\eta$, the transverse momentum starts suppressing as $\eta$ increases and hence the balance energy increases with increase in $\eta$. (iii) the variation in $\left\langle P_{x}^{\text {dir }}\right\rangle$ with $\eta$ decreases with increase in impact parameter. But with increase in impact parameter the balance energy increases for all values of $\eta$.

The above mentioned findings can be understood by decomposing the total transverse momentum into contributions due to mean field and two- body nn collisions as shown in Fig. 4 . The symbols are explained in the caption of the figure. One notices that mean field flow increases up to couple of hundred $\mathrm{MeV} /$ nucleon and then saturates. In the lower incident energy region, the flow due to mean field is smaller for larger asymmetries compared to smaller asymmetries. At higher incident energies the mean field flow is nearly independent of $\eta$. Similar behavior is seen 


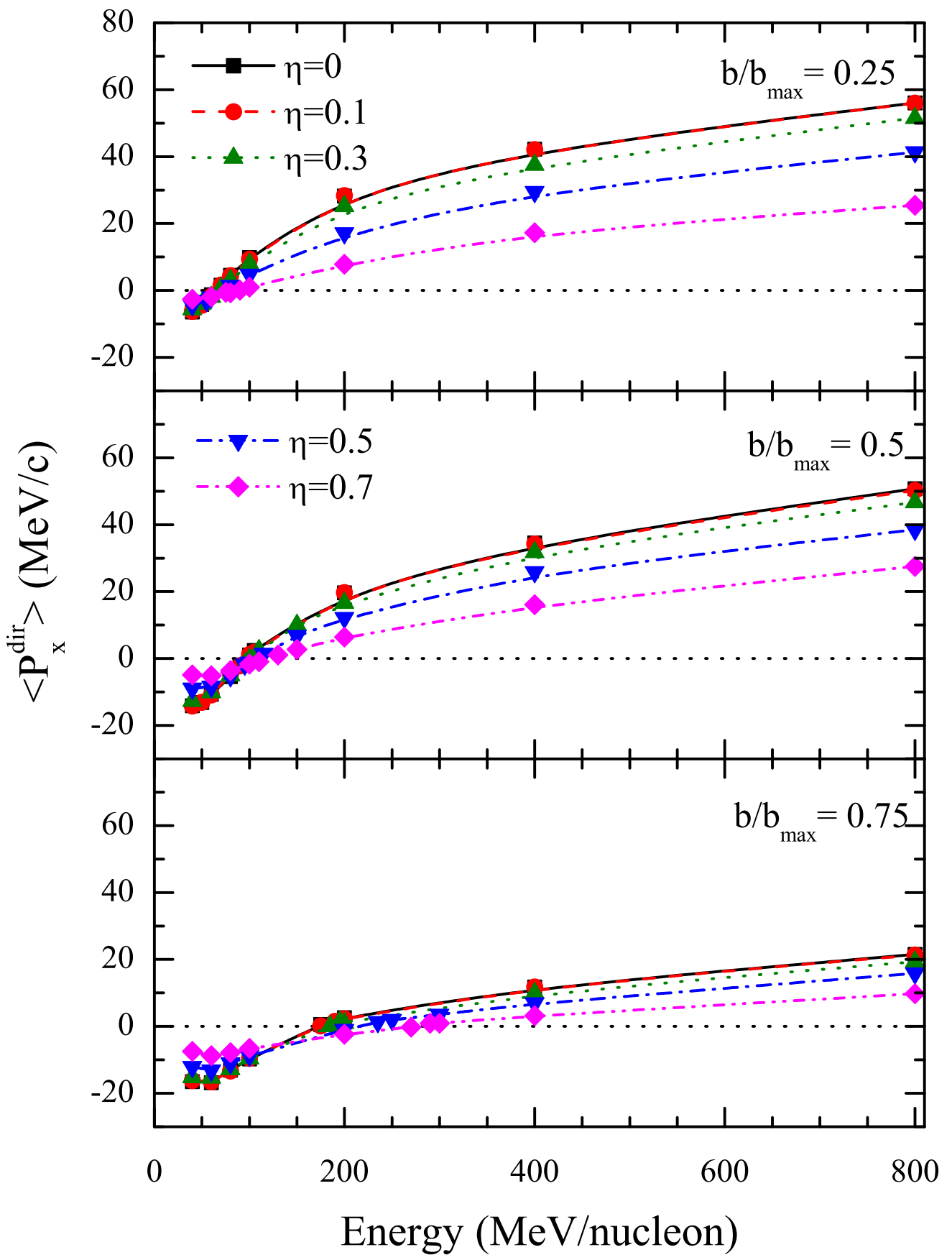

Figure 3. (Color online) The $\left\langle P_{x}^{d i r}>(\mathrm{MeV} / \mathrm{c})\right.$ as a function of incident energy for system mass $A_{T O T}=240$. The results are shown for different mass asymmetries $(\eta=0-0.7)$ at reduced impact parameters $b / b_{\max }=0.25$ (upper panel), 0.5 (middle panel), and 0.75 (bottom panel). The lines are only to guide the eye. Symbols have the same meaning as in Fig. 1. 


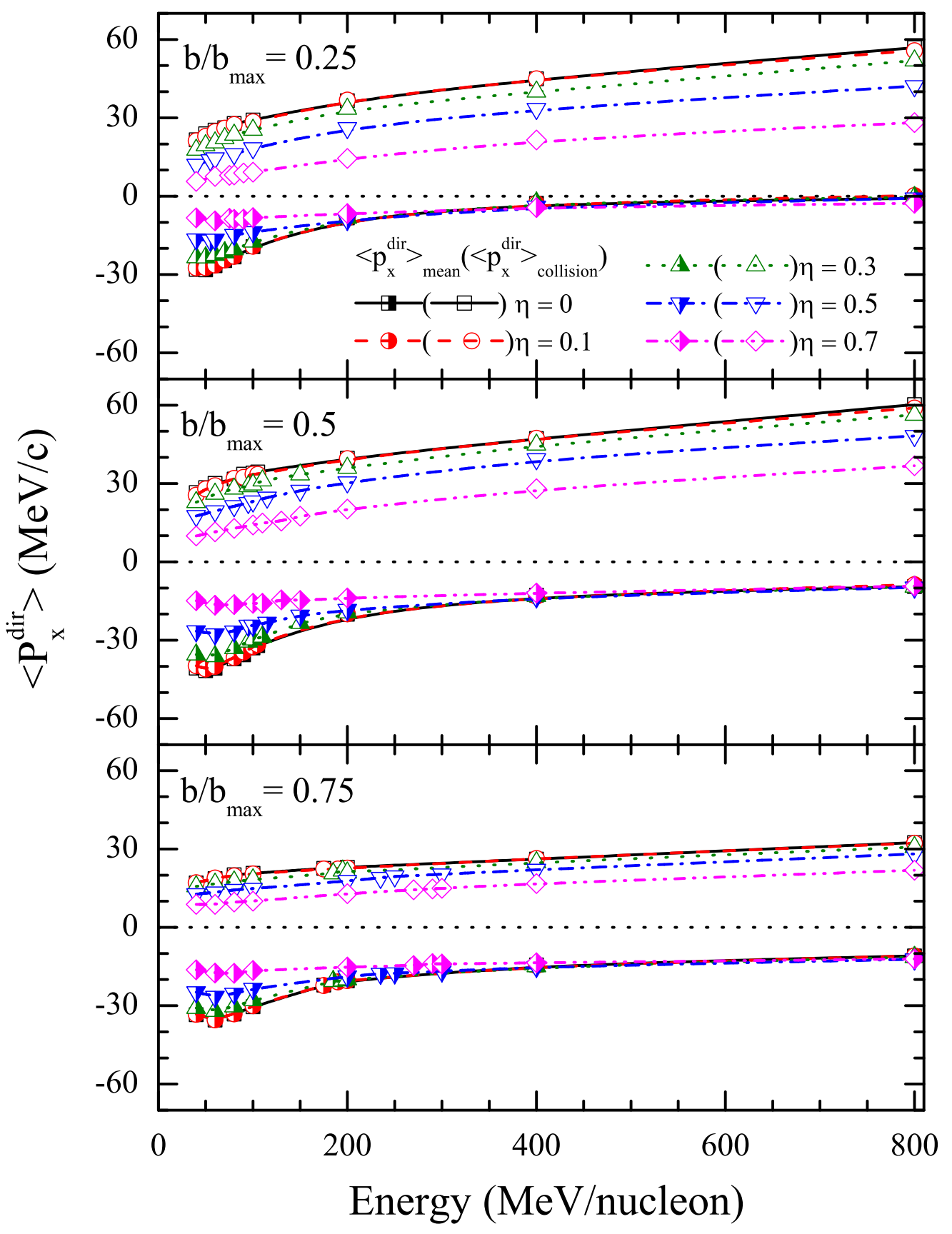

Figure 4. (Color online) The decomposition of $\left\langle P_{x}^{d i r}\right\rangle$ displayed in Fig. 3 into mean field (half filled symbols) and collision part (open symbols) as a function of incident energy. 


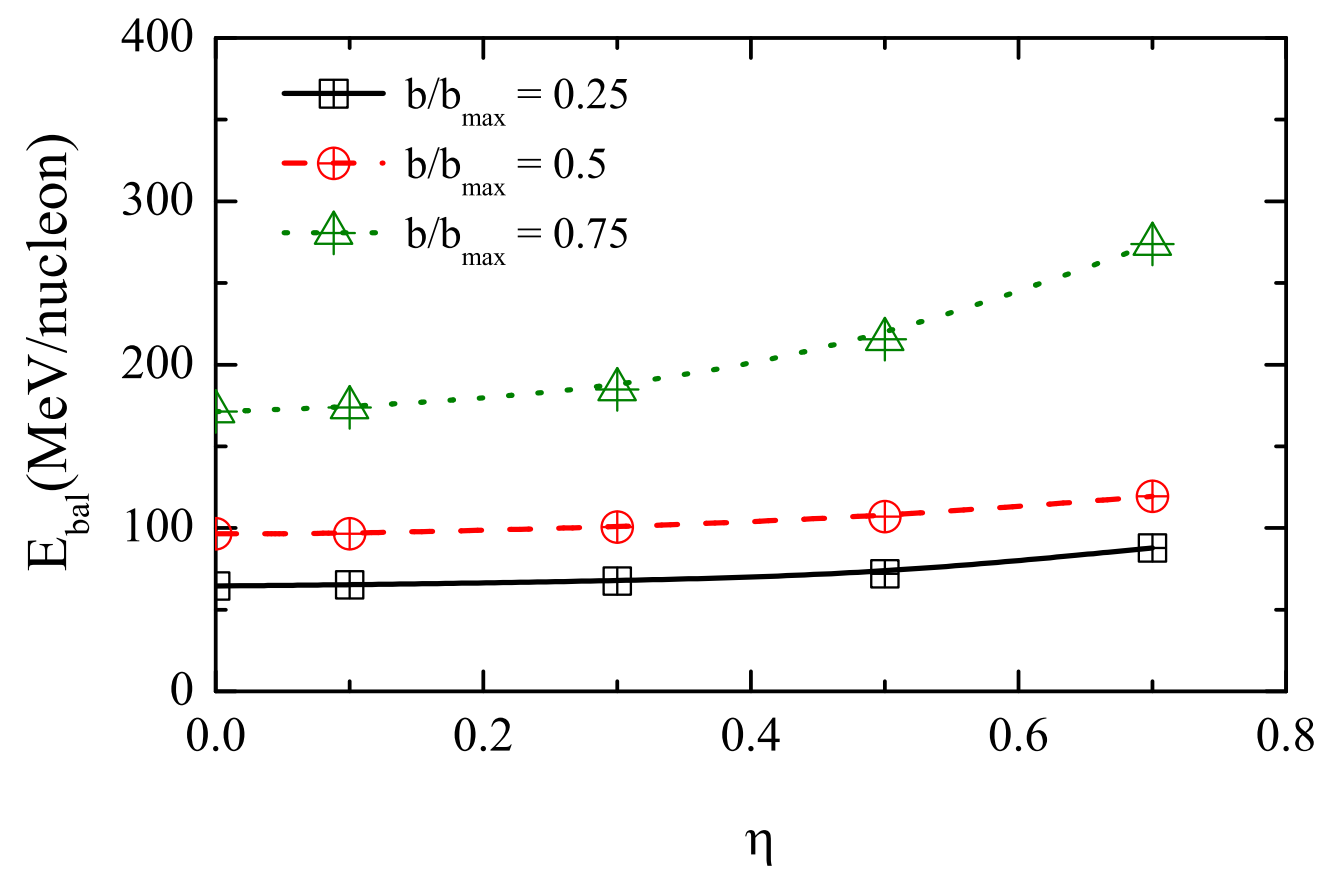

Figure 5. (Color online) The $E_{b a l}$ as a function of $\eta$ for system mass $A_{T O T}=240$. The results for different impact parameters $b / b_{\max }=0.25,0.5$, and 0.75 are represented, respectively, by the crossed squares, circles, and triangles. Lines are to guide the eye.

for other impact parameters also. The flow due to binary nn collisions, however, increases with increase in incident energy for all $\eta$, but as the impact parameter increases, the nn collision flow starts saturating. Obviously as explained earlier, the flow due to binary nn collisions decreases with increase in $\eta$.

In Fig. 5, we display $E_{b a l}$ as a function of $\eta$ for $b / b_{\max }=0.25,0.5$, and 0.75 , keeping $A_{T O T}$ fixed as 240. Various symbols are explained in the caption of the figure. From the figure, we see that $E_{b a l}$ increases with increase in $\eta$ for all values of $b / b_{\max }$. Also the $\eta$ dependence of $E_{b a l}$ increases with increase in impact parameter.

In Fig. 6, we display the percentage change in balance energy $\Delta E_{b a l}^{b / b_{\max }}(\%)$, defined as $\Delta E_{b a l}^{b / b_{\max }}(\%)=\left(\left(E_{b a l}^{b / b_{\max } \neq 0.25}-E_{\text {bal }}^{b / b_{\max }=0.25}\right) / E_{b a l}^{b / b_{\max }=0.25}\right) \times 100$ as a function of $\eta$. Lines represents the mean value of variation. It is clear from the figure that the effect of impact parameter variation is almost uniform throughout the asymmetry range for every fixed system mass. We also found that the mean variation decreases with increase in $A_{T O T}$. Clearly, the effect of impact parameter variation is independent of $\eta$.

In Fig. 7 , we display the percentage difference $\Delta E_{b a l}^{\eta}(\%)$ defined as $\Delta E_{b a l}^{\eta}(\%)=\left(\left(E_{b a l}^{\eta \neq 0}\right.\right.$ $\left.\left.E_{b a l}^{\eta=0}\right) / E_{b a l}^{\eta=0}\right) \times 100$ as a function of reduced impact parameter $\left(b / b_{\max }\right)$. Lines are the linear fits $\left(\propto m \frac{b}{b_{\max }}\right)$. It is clear from the figure that the effect of the asymmetry variation increases with increase in the impact parameter for each mass range. This is due to the fact that with increase in impact parameter, the number of binary nn collisions decreases and the increase of mass asymmetry further adds the same effect. 


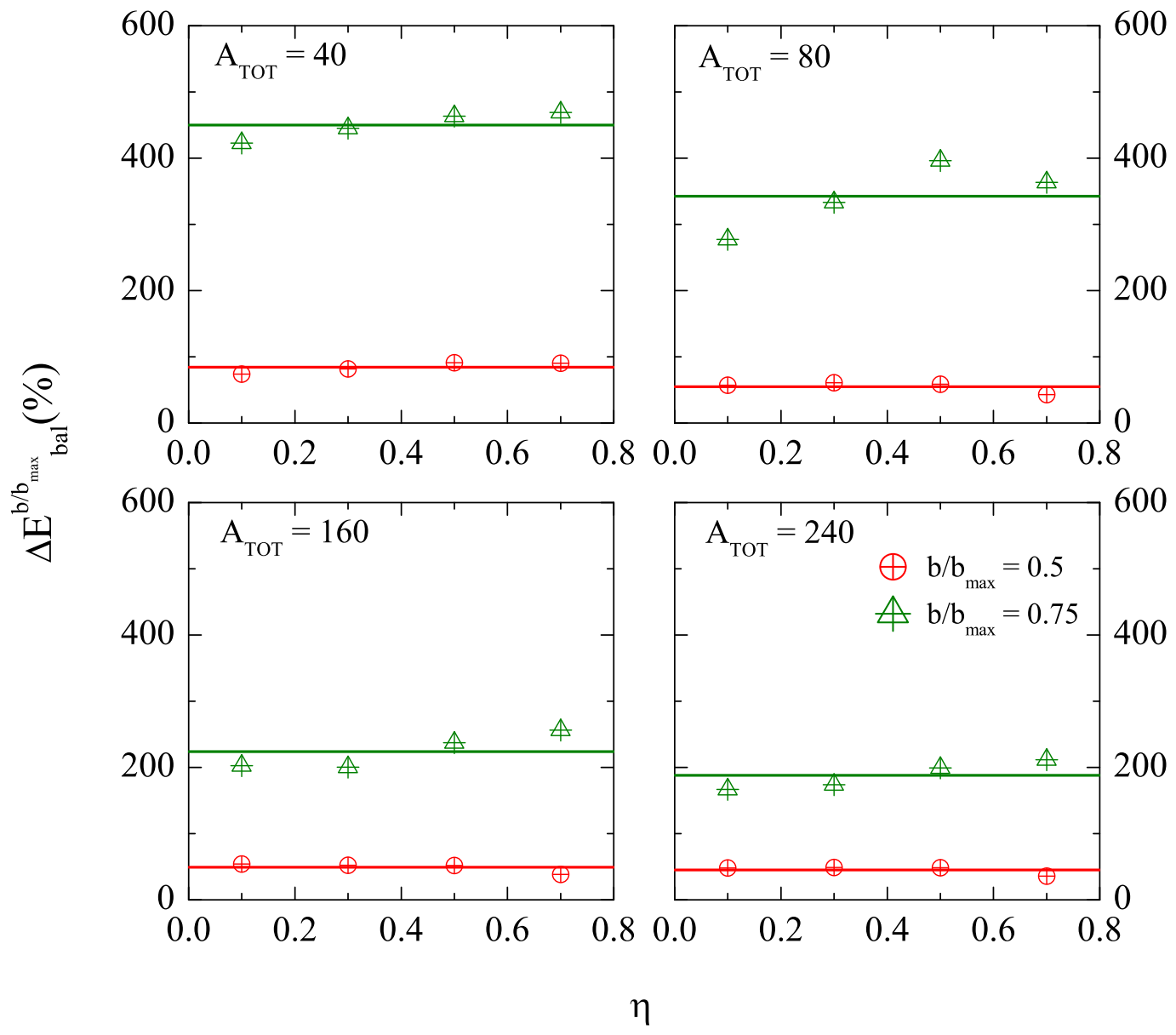

Figure 6. (Color online) The percentage difference $\Delta E_{b a l}^{b / b_{\max }}(\%)$ as a function of $\eta$ for different system masses. The results of the percentage difference for different colliding geometries $b / b_{\max }$ $=0.5$ and 0.75 are represented, respectively, by the crossed circles and triangles. Horizontal lines represent the mean value of $\Delta E_{b a l}^{b / b_{\max }}(\%)$ for each $b / b_{\max }$.

\section{Summary}

We presented a detailed study on the role of impact parameter on the collective flow and its disappearance for different mass asymmetric reactions using the quantum molecular dynamics model. For the present study, the mass asymmetry of the reaction is varied from 0 to 0.7 by keeping the total mass of the system fixed. A significant role of impact parameter on the collective flow and its disappearance for the mass asymmetric reactions is seen. The impact parameter dependence is also found to vary with mass asymmetry of the reaction.

\section{Acknowledgments}

Author is thankful to Council of Scientific and Industrial Research (CSIR) for providing the Junior Research Fellowship.

\section{References}

[1] Krofcheck D et al. 1989 Phys. Rev. Lett. 632028 


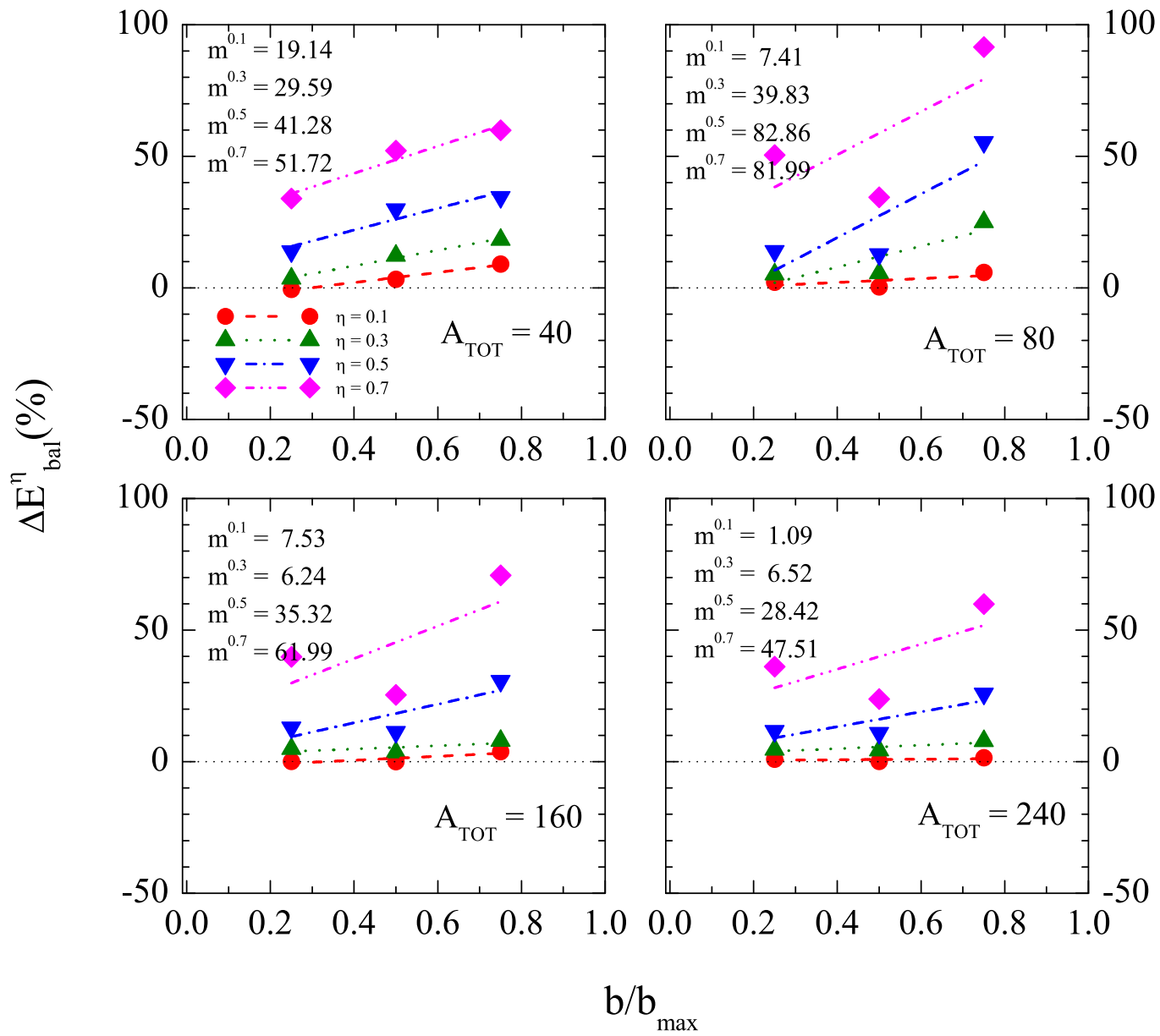

Figure 7. (Color online) The percentage difference $\Delta E_{b a l}^{\eta}(\%)$ as a function of $b / b_{\max }$ for different system masses. The results of the percentage difference for different asymmetries $\eta=0.1,0.3$, 0.5 , and 0.7 are represented, respectively, by the solid circles, triangles, inverted triangles, and diamonds. Lines are the linear fits $\left(\propto \frac{m}{b_{\max }}\right) ; m$ values without errors are displayed.

[2] Lukasik J et al. 2005 Phys. Lett. B 608223

[3] Mota la V de et al. 1992 Phys. Rev. C 67746

Zhou H, Li Z and Zhuo Y 1994 Phys. Rev. C 50 R2664

Cussol D et al. 2002 Phys. Rev. C 65044604

[4] Westfall G D et al. 1993 Phys. Rev. Lett. $\mathbf{7 1} 1986$

[5] Pak R et al. 1996 Phys. Rev. C 53 R1469

[6] Magestro D J et al. 2000 Phys. Rev. C 61 021602(R)

[7] Magestro D J et al. 2000 Phys. Rev. C 62 041603(R)

[8] Sood A D and Puri R K 2004 Phys. Lett. B 594260

Sood A D and Puri R K 2004 Phys. Rev. C 69054612

Sood A D and Puri R K 2006 Phys. Rev. C 73067602

Sood A D and Puri R K 2006 Eur. Phys. J. A 30571

[9] Sullivan J P et al. 1990 Phys. Lett. B 2498

[10] Buta A et al. 1995 Nucl. Phys. A 584397

[11] Goyal S and Puri R K 2011 Nucl. Phys. A 853164

[12] Hong B et al. 2000 Phys. Rev. C 66034901

[13] Chugh R and Puri R K 2010 Phys. Rev. C 82014603 
[14] Chugh R and Puri R K 2010 Int. J. Mod. Phys. E 192009

[15] Huang S W et al. 1993 Phys. Lett. B 29841

Huang S W et al. 1993 Prog. Nucl. Part. Phys. 30105

Lehmann E et al. 1993 Prog. Nucl. Part. Phys. 30219

Batko G et al. 1994 J. Phys. G 20461

Lehmann E et al. 1995 Phys. Rev. C 512113

Fucks C 1996 J. Phys. G 22131

Puri R K et al. 1996 Phys. Rev. C 5428

Kumar S et al. 1998 Phys. Rev. C 583494

Hartnack C et al. 1998 Eur. Phys. J. A 1151

Kumar S et al. 1998 Phys. Rev. C 572744

Puri R K et al. 2000 J. Comp. Phys. 162245

Sood A D and Puri R K 2004 Phys. Rev. C 70034611

Dhawan J K et al. 2007 Phys. Rev. C 75057610

Kumar S et al. 2008 Phys. Rev. C 78064602

Sood A D et al. 2009 Phys. Rev. C 79064618

Vermani Y K et al. 2009 Eur. Phys. Lett. 85062001

Vermani Y K et al. 2009 J. Phys. G 36105103

Sood A D et al. 2010 Phys. Rev. C 82014603

Kumar S et al. 2010 Phys. Rev. C 81014611

Kumar S et al. 2010 Phys. Rev. C 81014601

Vermani Y K et al. 2010 J. Phys. G 37015105

Gautam S et al. 2010 J. Phys. G 37085102

Gautam S et al. 2011 Phys. Rev. C 83014603

Gautam S et al. 2011 Phys. Rev. C 83034606

Hauer W et al. 2011 Phys. Lett. B 697512

Goyal S and Puri R K 2011 Phys. Rev. C 83047601

[16] Aichelin J 1991 Phys. Rep. 202233

[17] Puri R K et al. 1991 Phys. Rev. C 43315

Puri R K et al. 1997 Phys. Rev. C 451837

Puri R K et al. 1998 Eur. Phys. J. A 3277

Puri R K et al. 2000 Eur. Phys. J. A 8103

Puri R K et al. 2005 Eur. Phys. J. A 23429

Dutt I et al. 2010 Phys. Rev. C 81044615

Dutt I et al. 2010 Phys. Rev. C 81047601

Dutt I et al. 2010 Phys. Rev. C 81064609

Dutt I et al. 2010 Phys. Rev. C 81064608

Kumar S et al. 2010 Chinese Phys. Lett. 27062504

[18] Chugh R and Sood A D 2011 arXiv:1010.1120v2 